\title{
Efecto del consumo de Leucaena leucocephala sobre la carga parasitaria de terneros destetados con pastoreo directo
}

\author{
Figueroa, C. ${ }^{1}$; Mancebo, O. ${ }^{2}$; Scribano, V. ${ }^{3}$; Rigonatto, T. ${ }^{4}$ \\ ${ }^{1}$ INTA-IPAF Región NEA. ${ }^{2}$ CIT-Formosa y Cátedra Sanidad Animal FRN-UNAF. \\ ${ }^{3}$ INTA Laguna Blanca. ${ }^{4}$ Cátedra Enfermedades Parasitarias Fac.Cs.Veterinarias, \\ UNNE, Corrientes, Argentina. E-mail: trigonatto@vet.unne.edu.ar
}

\section{Resumen}

Figueroa, C.; Mancebo, O.; Scribano, V.; Rigonatto, T.: Efecto del consumo de Leucaena leucocephala sobre la carga parasitaria de terneros destetados con pastoreo directo. Rev. Vet. 30: 2, XX-XX, 2019. Con el objeto de evaluar el efecto del consumo de Leucaena sp sobre la carga parasitaria gastrointestinal de terneros destetados, se llevó a cabo un ensayo durante 105 días en el departamento Pilagás (Formosa, Argentina). Se seleccionaron 30 terneros y se los dividió en dos lotes: 15 terneros con Leucaena (TRAT c/L) y 15 sin Leucaena (TRAT s/L). El ensayo se desarrolló en un sistema de pastoreo directo donde los animales fueron infestados naturalmente. El lote TRAT c/L pastoreó en una intersiembra de L. leucocephala con Brachiaria brizantha. El grupo TRAT s/L, lo hizo sólo con B. brizantha. Se determinó la cantidad de huevos de parásitos por gramo (HPG), cultivo e identificación de larvas, valores de hematocrito (Hto) y peso vivo. Los principales géneros recuperados en el coprocultivo fueron: Haemonchus y Trichostrongylus, siguiendo en orden decreciente Bunostomum, Oesophagostomum, Cooperia y Strongyloides. A partir del segundo muestreo, los animales que presentaron valores de Hto $\leq 22 \%$ debieron ser tratados con albendazol ya que estaba comprometido su estado de salud. Durante el ensayo un solo ternero del TRAT c/L debió ser tratado, mientras que 9 individuos correspondientes al TRAT s/L requirieron tratamiento. En cuanto a la evolución de HPG, se observaron diferencias significativas en el segundo y cuarto muestreo (p valor de 0,3 y 0,006 respectivamente) a favor del TRAT s/L. Se concluye que bajo las condiciones de la presente experiencia, los resultados demostraron un efecto del consumo de L. leucocephala sobre la disminución de HPG, lo que amerita la realización de futuras experiencias para un mejor conocimiento de la alternativa de control integrado de estos parásitos.

Palabras clave: terneros, nematodos gastrointestinales, Leucaena leucocephala, reducción de huevos/gramo.

Recibido: febrero 2019 / Aceptado: abril 2019

\begin{abstract}
Figueroa, C.; Mancebo, O.; Scribano, V.; Rigonatto, T. Effect of feed intake of Leucaena leucocephala on gastrointestinal parasitic load of weaned calves. Rev. Vet. 30: 2, $X X-X X, 2019$. The present study was carried out during 105 days in Pilagás (Formosa, Argentina) to evaluate the effect of Leucaena sp consumed by weaned calves on the gastrointestinal parasitic load. Thirty calves were divided into two groups: grazing Leucaena (TREATED with/L; $\mathrm{n}=15$ ) or without consumption of Leucaena (TREATED without/L; $n=15$ ). This essay was performed in a direct grazing system and animals
\end{abstract}


were naturally infected under the conditions of Pilagás forage. Animals in the TREATED with/L group grazed in an interseeding of Leucaena leucocephala with Brachiaria brizantha while the TREATED without/L group grazed only on B. brizantha. Eggs per gram (EPG) were determined from feces. Fecal culture and identification of larvae were also performed for each experimental group. Hemotocrit $(\mathrm{Ht})$ was measured and animals were weight. The main genera recovered in the fecal samples were: Haemonchus, Trichostrongylus, and Bunostomum. Oesophagostomum, Cooperia and Strongyloides were less abundant. Since the second sampling, animals with $\mathrm{Ht} \leq 22 \%$ required albendazole administration since health was compromised. During the whole study, only one calf under TREATED with/L required treatment, whereas in the other group (TREATED without/L) nine animals needed treatment. In relation to EPG, significant differences were registered for the second and forth sampling ( $\mathrm{p}$ value of 0.3 and 0.006, respectively) in favor of TREATED without/L group. We conclude that under the conditions for the present study, L. leucocephala intake exerts an effect over EPG. Therefore, future assays should be performed for a better understanding regarding an alternative integral control of parasites.

Key words: calves, gastrointestinal nematodes, Leucaena leucocephala, reduction of eggs/gram.

\section{INTRODUCCIÓN}

En Argentina, al igual que en la mayor parte del mundo, durante los últimos 30 años los antihelmínticos se convirtieron en insumos de producción muy eficientes y seguros. Sin embargo, el desarrollo de nematodos resistentes a tales drogas se ha transformado en una seria amenaza al dificultar la reducción de los costos inherentes al control del parasitismo ${ }^{4}$.

La resistencia antihelmíntica se define básicamente como la disminución de eficacia de un producto frente a poblaciones parasitarias que normalmente -a una dosis determinada- eran susceptibles al mismo ${ }^{26}$. Ello puede ser consecuencia de una modificación genética o de un incremento en la frecuencia de expresión de un carácter hereditario, pero en ambos casos los nematodos que sobreviven al tratamiento van a transmitir estos alelos resistentes a su progenie ${ }^{4}$.

La prevalencia de tal resistencia es particularmente alta en los pequeños rumiantes de Argentina, pero también se constata en bovinos y equinos ${ }^{3}$. Un estudio nacional realizado en 2005 demostró que de 69 rodeos bovinos, el 60\% presentaba fallas para el control de los nematodos utilizando ivermectinas o benzimidazoles ${ }^{8}$.

Particularmente en el área centro-norte de Argentina, la situación parece estar agravándose en los últimos años, especialmente en la Provincia de Corrientes ${ }^{4}$, en la cual se incrementó rápidamente desde los estudios realizados en 2005, donde del total de los rodeos estudiados $(n=8)$ el $75 \%$ eran resistentes a alguna droga, hasta el último realizado en 2014 donde el $100 \%$ de los rodeos $(n=4)$ presentaba resistencia ${ }^{15}$.

El aumento de la resistencia antihelmíntica, el costo, los problemas ligados a la toxicidad, la contaminación del medio ambiente y los residuos en los productos de origen animal ${ }^{22}$ hacen que las tendencias actuales se orienten hacia la integración de varios métodos de control ${ }^{27}$. Ellos incluyen el manejo de pastizales con pastoreo rotativo y mixto entre diferentes especies ${ }^{5}$, la selección genética de animales resistentes al parasitismo, con una mayor utilización de las razas autóctonas ${ }^{2,18}$, controles biológicos ${ }^{23}$, método Famacha ${ }^{29}$ y tratamientos selectivos con poblaciones refugio ${ }^{14,19}$. 
Dentro de estas nuevas estrategias, se han comenzado a evaluar las potencialidades de algunas sustancias presentes en los forrajes, denominadas metabolitos secundarios de plantas. Entre los más estudiados se encuentran los taninos, compuestos polifenólicos sintetizados en forma natural por los vegetales. Estos poseen una amplia distribución en diversas plantas y semillas, así como elevada actividad biológica ${ }^{27}$.

Existen dos tipos de taninos: los hidrolizables y los condensados (TC) ${ }^{11}$, siendo éstos últimos, en el campo de la nutrición animal, el grupo de compuestos mejor estudiados en cuanto a su repercusión fisiológica y su amplia distribución ${ }^{13}$.

Los TC se asocian como parte de la defensa de las plantas contra insectos y herbívoros $^{13}$. Diversas investigaciones han comprobado que el consumo de plantas con contenidos moderado de TC puede tener un efecto directo en la disminución de los nematodos gastrointestinales ${ }^{6,12} \mathrm{o}$ un efecto indirecto a través de la absorción de la proteína bypass en el intestino delgado, lo cual fortalecería el sistema inmunitario y mejoraría la resistencia o resiliencia de los animales frente a infestaciones parasitarias ${ }^{5,30}$.

Concentraciones de TC de 6-12\% de materia seca en el forraje, deprimen el consumo y la digestibilidad del mismo, mientras que a concentraciones de $2-4 \%$, disminuyen la proteólisis de los microorganismos del rumen y por tanto incrementan la absorción intestinal de las proteínas y contribuyen a mejorar la salud de los animales al reducir los efectos perjudiciales de los parásitos internos ${ }^{1}$.

Se ha comprobado que algunas leguminosas, tanto arbóreas como forrajeras tradicionales, presentan las concentraciones adecuadas de TC para producir un efecto antihelmíntico. Las plantas con estas características disminuyen las infestaciones parasitarias en los animales y, además, contribuyen a mejorar el plano nutricional ${ }^{10}$.

Leucaena leucocephala es un árbol tropical que desde hace tiempo despertó el interés mundial por sus atributos como recurso forrajero, y que se desarrolla adecuadamente en el norte y centro de Argentina. Puede alcanzar alturas de entre 7 y 12 metros, sus hojas son compuestas, de 9 a $25 \mathrm{~cm}$ de largo, verde grisáceas y sin pelos. Evoluciona muy bien con precipitaciones anuales entre 850 y $1500 \mathrm{~mm}$, sin embargo crece desde sitos secos (350 mm/año) hasta muy húmedos (3000 mm/año), con temperaturas de 20 a $30^{\circ} \mathrm{C}^{24}$.

Sus hojas son ricas en proteínas fácilmente digeribles por los rumiantes. La calidad del forraje es similar a la de la alfalfa, aunque es más abundante en betacarotenos y proteína by-pass, por lo cual disminuyen los riesgos de meteorismo (empaste), y en general tiene alta palatabilidad ${ }^{24}$.

Estudios in vitro realizados en 2011 con extracto de Leucaena han confirmado que tiene efecto antihelmíntico al inhibir el proceso de eclosión de huevos y reducir el establecimiento de larvas L3 al afectar el desenvainamiento ${ }^{16,21}$. Sin embargo, deben realizarse más estudios in vivo para evaluar su toxicidad y actividad antihelmíntica.

Por la disponibilidad en la región, su elevado valor nutritivo y su probada acción antihelmíntica, el presente trabajo se propuso investigar el efecto del consumo de Leucaena sobre la carga parasitaria gastrointestinal en terneros destetados en condiciones de pastoreo directo.

\section{MATERIAL Y MÉTODOS}

Región geográfica. El ensayo se llevó a cabo en la localidad de Tres Lagunas, departamento Pilagás, Provincia de Formosa, ubicada a los $25^{\circ} 11^{\prime} 09^{\prime \prime}$ de latitud sur y $58^{\circ}$ $32^{\prime}$ 52” de longitud oeste, a una altitud de 86 msnm, valores medios anuales de 1.100 a $1.300 \mathrm{~mm}$ de precipitaciones y $21,9^{\circ} \mathrm{C}$ de temperatura. 
Sistema de pastoreo. Se desarrolló en un sistema de pastoreo directo, en el que se utilizaron terneros recientemente destetados, criollos cruza con Brangus y Braford, de ambos sexos, de 8 a 10 meses de edad, con un promedio de $153 \mathrm{~kg}$ de peso vivo. Con el objetivo de establecer el área de los potreros, la oferta forrajera se estimó para una carga de 15 terneros de $180 \mathrm{~kg}$ por 180 días.

Terneros. Una semana previa al inicio del ensayo, se tomaron muestras de materia fecal en 60 terneros para medir el grado de infestación por nematodos gastrointestinales a través del recuento de huevos por gramo (HPG) en heces. A partir de estos resultados, se seleccionaron 30 terneros y se dividieron en 2 lotes de 15 animales, de forma que estos dos grupos fuesen homogéneos en cuanto a sexo, peso y carga parasitaria.

Tratamientos. El ensayo se realizó en dos potreros contiguos, separados por un alambrado convencional, a cada uno de los cuales se le asignó un lote de animales y un tratamiento. Para mejor uso de la pastura, cada potrero se subdividió con alambrado eléctrico. El lote de animales del tratamiento con Leucaena (TRAT c/L) se ubicó en un potrero con una superficie de 1,8 ha, sembrado con Leucaena leucocephala con una intersiembra de Brachiaria brizantha cv marandú. El lote del tratamiento sin Leucaena (TRAT s/L) se dispuso en un potrero de 2 ha sembrado con $B$. brizantha cv marandú; la dieta de estos animales se complementó con semillas de algodón, como suplemento proteico, a razón de 500 g/cabeza/día.

Muestreos. El pastoreo sobre ambos lotes se inició a comienzos del mes de mayo y cada 21 días se realizaron muestreos, durante 105 días. Por lo tanto, se efectuaron 5 muestreos secuenciales durante la experiencia. Debido al estado general que presentaban los animales, en el segundo muestreo se decidió incorporar análisis de sangre al set de datos.

Exámenes coproparasitológicos. Las muestras de materia fecal fueron extraídas directamente del recto, identificadas y enviadas al laboratorio para realizar exámenes coprológicos: HPG mediante la técnica de MacMaster modificada ${ }^{25}$ e identificación de larvas a través de coprocultivo por el método Corticelli y Lai ${ }^{9}$.

Controles hematológicos. Se tomaron muestras de sangre obtenidas de la vena yugular para determinar la valoración de hematocrito (Hto) a través de la técnica del microhematocrito y extendido de sangre coloreado con Giemsa para descartar infección por Anaplasma sp, Babesia sp y Trypanosoma sp. Los análisis de laboratorio (HPG, coprocultivo, Hto y extendido de sangre) fueron realizados por en el Centro de Investigaciones y Transferencia (CIT) de Formosa. Los animales que presentaron sintomatología de la enfermedad con altos valores de HPG y descenso de Hto $(\leq$ a $22 \%$ ) fueron tratados con antiparasitarios (albendazol $10 \%$ a dosis de $10 \mathrm{mg} / \mathrm{kg}$ ) por verse comprometida su salud.

Análisis estadísticos: Inicialmente los dos grupos experimentales contaban con 15 terneros cada uno. El grupo correspondiente al TRAT s/L perdió 9 unidades experimentales (en diferentes momentos a lo largo del ensayo) por la necesidad de aplicar tratamientos antiparasitarios. El grupo correspondiente al TRAT c/L perdió 2 unidades experimentales, uno por un accidente en la manga y otro por necesidad de tratamiento 
antiparasitario. Si bien el registro de datos se le efectuó a todos los animales durante el ensayo, los valores de los terneros que recibieron antiparasitarios no fueron incluidos en los análisis estadísticos de los momentos posteriores a esa aplicación. En diferentes etapas de la experiencia, algunas variables no pudieron ser evaluadas, por lo cual el número de repeticiones varía. Los datos relevados fueron analizados con el programa estadístico Infostat y se sometieron al análisis de la varianza con test de Duncan y prueba F, así como al análisis de componentes principales.

\section{RESULTADOS Y DISCUSIÓN}

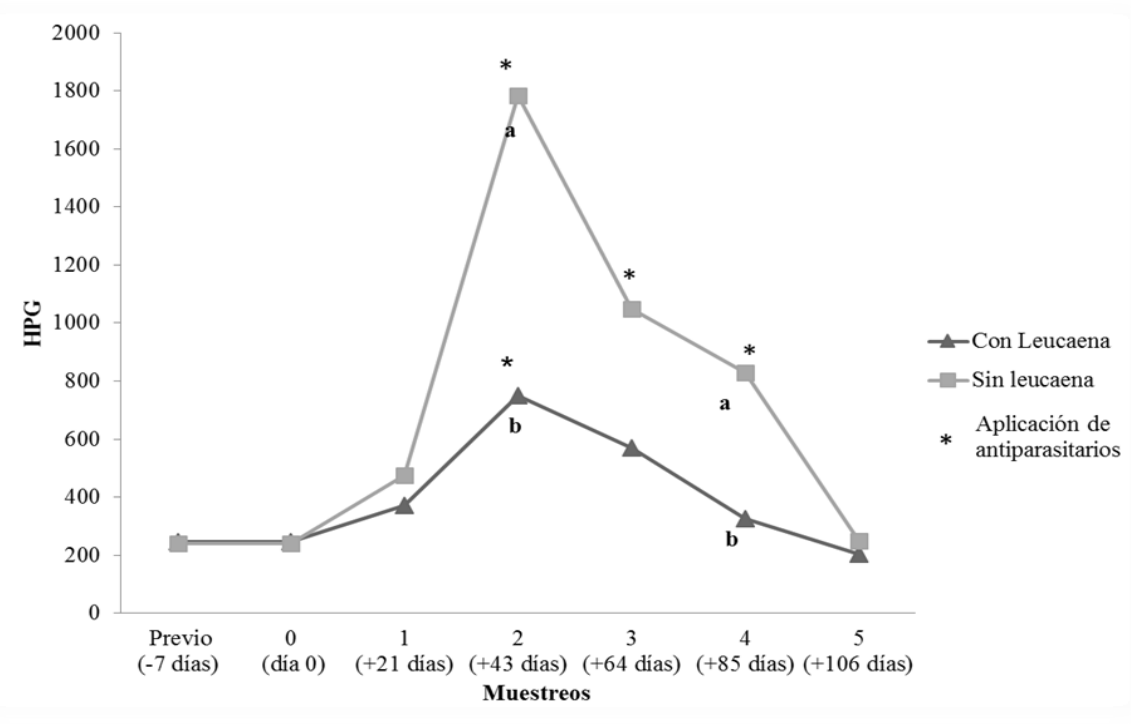

Figura 1. Evolución de HPG según tratamiento, en diferentes momentos de medición. Letras diferentes indican diferencias significativas entre tratamientos.

Recuento de huevos (Figura 1). Los resultados de HPG (prueba F con p valor 0,05 ) demuestran que en la segunda y cuarta medición existieron diferencias significativas a favor del TRAT c/L. Resultados similares se observaron en estudios realizados con terneras para evaluar el efecto de la suplementación con extracto de quebracho, donde hubo una reducción significativa de HPG en los individuos suplementados ${ }^{17}$.

También se obtuvieron resultados favorables en pequeños rumiantes que consumieron pasturas taníferas, los cuales registraron recuentos de huevos fecales más bajos en comparación con los animales que pastaron en forrajes de bajo contenido de TC ${ }^{7,20}$.

Durante el primer y tercer momento de muestreo, no se registraron diferencias significativas entre tratamientos; no obstante los valores del TRAT s/L duplicaron y triplicaron respectivamente los resultados del TRAT c/L. La equiparación de los valores a medida que discurrían los muestreos se atribuye a que los animales con valores mayores de HPG (que correspondieron al grupo sin Leucaena) fueron eliminados del análisis al ser desparasitados en los distintos momentos de las mediciones.

Coprocultivo (Figura 2). Los géneros identificados en los coprocultivos para ambos lotes (Figura 1) fueron principalmente Haemonchus sp y Trichostrongylus sp, siguiendo en orden decreciente, Bunostomum sp, Oesophagostomum sp, Cooperia sp y 
Strongyloides sp, donde Haemonchus y Trichostrongylus representaron el $70 \%$ de las larvas identificadas en todos los muestreos. Resultados similares se encontraron en otros estudios realizados en Sudamérica ${ }^{8,28}$. En los exámenes de los extendidos coloreados con Giemsa no se observaron hemoparásitos (Anaplasma sp, Babesia sp ni Trypanosoma sp), por lo cual se descartó la ocurrencia de enfermedades anemizantes.
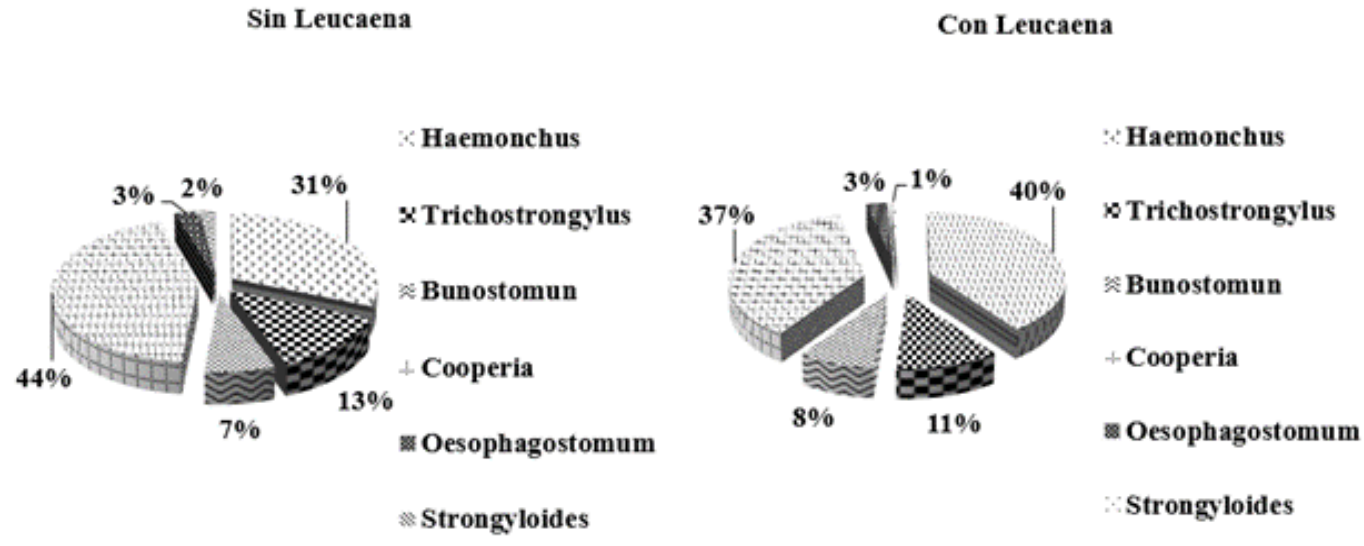

Figura 2. Resultados promedios de los coprocultivos de ambos tratamientos.

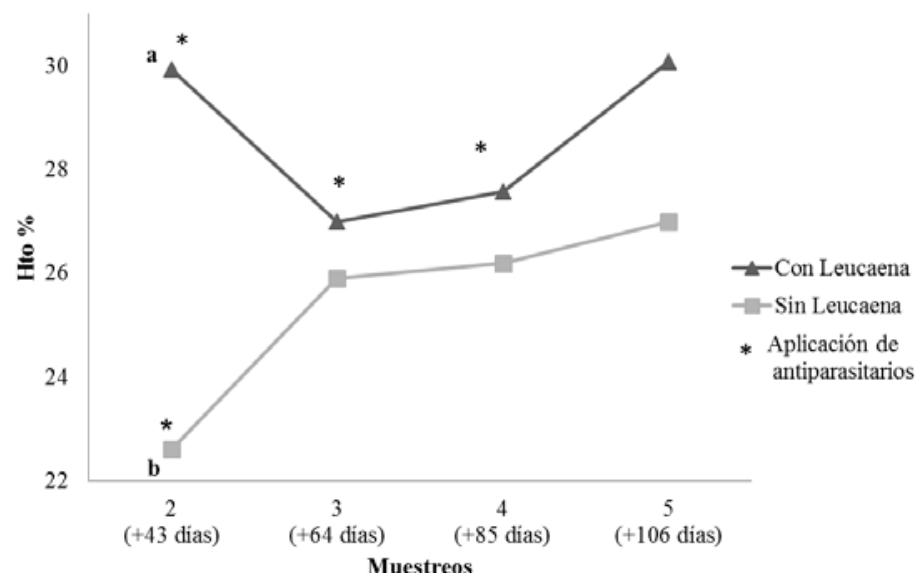

Figura 3. Evolución del hematocrito según tratamientos. Letras diferentes indican diferencias significativas entre tratamientos.

Hematocrito (Figura 3). Los valores de Hto analizados (prueba F con p valor $0,05)$ sólo mostraron diferencias significativas entre tratamientos en el segundo momento muestral. A pesar de no existir diferencias en los demás momentos de la experiencia, el TRAT c/L siempre presentó valores más altos que el lote sin Leucaena. Los animales que recibieron la aplicación de antiparasitario, y que por tanto fueron excluidos de los análisis estadísticos, siguieron siendo muestreados. Los valores de Hto de estos terneros mostraron una rápida recuperación tras la aplicación de antiparasitarios, lo cual indica que la causa de esos bajos valores fue efectivamente la parasitosis.

Tabla 1. Análisis de pesos y ganancia media diaria (GMD) por tratamientos, en los diferentes momentos de la medición. 


\begin{tabular}{cccc}
\hline & & \multicolumn{2}{c}{ tratamientos } \\
\cline { 3 - 4 } muestreos & pesos & con Leucaena & sin Leucaena \\
\hline 1 & peso promedio $(\mathrm{kg})$ & $151,57 \pm 34,41$ & $153,6 \pm 27,80$ \\
$(+21$ días $)$ & $\mathrm{n}$ & 15 & 15 \\
2 & peso promedio $(\mathrm{kg})$ & $159,32 \pm 34,37$ & $140 \pm 21,18$ \\
$(+42$ días $)$ & GMD en g/an/día & $348,64 \mathrm{a}$ & $-647,62 \mathrm{a}$ \\
3 & $\mathrm{n}$ & 14 & 15 \\
$(+63$ días $)$ & peso promedio $(\mathrm{kg})$ & $155,75 \pm 50,06$ & $140,5 \pm 21,18$ \\
& GMD en g/a/día & $-177,66 \mathrm{a}$ & $-185,71 \mathrm{a}$ \\
5 & $\mathrm{n}$ & 13 & 10 \\
$(+105$ días $)$ & GMD en gramos/día & $-326,01 \mathrm{a}$ & $-373,02 \mathrm{a}$ \\
& $\mathrm{n}$ & 13 & 6 \\
diferencia & & $-2,58 \mathrm{a}$ & $-18,92 \mathrm{~b}$ \\
PF-PI & & $(-24$ g/an/día $)$ & $(-180,16$ g/an/día $)$ \\
\hline PF-PI: medias de peso final peso inicial $;$ n: número; g/a: gramos por animal
\end{tabular}

PF-PI: medias de peso final - peso inicial.; n: número; g/a: gramos por animal.

Pesajes (Tabla 1). El análisis de datos de los pesajes indica que no hubo diferencias significativas de los pesos promedio, ni de las GMD en ninguno de los muestreos. Sin embargo, en la diferencia de peso total hubo significación estadística (ANOVA, test de Duncan $\mathrm{p}=0,05)$ a favor del TRAT c/L. Hasta el segundo muestreo (+42 días) se observó que los TRAT c/L tuvieron en promedio un aumento de 7,75 kg (348,64 g/an/día) y que el lote sin Leucaena perdió en promedio 13,6 kg (647,62g/an/día).

En el tercer muestreo de ambos tratamientos hubo disminución de peso, pero en el TRAT c/L siempre se obtuvieron valores superiores al TRAT s/L. La equiparación de los valores a medida que discurrieron los muestreos se debió a que los animales que recibieron la aplicación de antiparasitarios (los que presentaron menores ganancias de peso vivo) fueron excluidos del análisis estadístico al ser desparasitados en los distintos momentos de las mediciones.

Análisis de componentes principales (Figura 4). Este estudio permitió reducir la dimensión analítica a dos componentes, conservando el 85,4\% de la variabilidad total (55,1\% en el primer componente y 30,3\% en el segundo).

El primer componente permite separar ambos tratamientos. A la derecha del biplot se ubican las unidades experimentales del TRAT s/L, tanto machos como hembras (M:s/L y H:s/L), asociados con los mayores valores de HPG en todas las mediciones y los menores valores de las demás variables. A la izquierda se encuentran las unidades experimentales del TRAT c/L, tanto machos como hembras (M:c/L y H:c/L), asociados a los mayores valores de Hto y pesos en todos los momentos de medición y a los menores valores de HPG. 


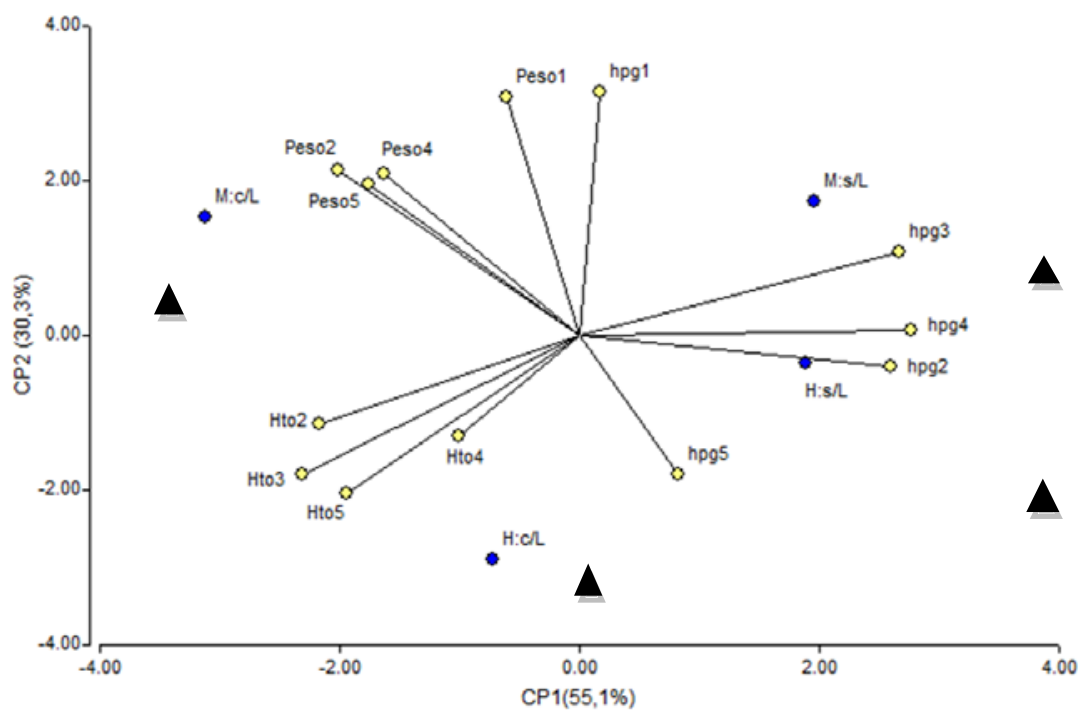

Figura 4. Diagrama de dispersión ("biplot”) que muestra la distribución conjunta de las variables (análisis de componentes principales = tratamientos y sexos).

El segundo componente, representado en el biplot sobre el eje vertical, permite separar los machos y las hembras del TRAT c/L. Los machos en la parte superior, se asocian a mayores pesos y menores Hto y las hembras en la parte inferior, se vinculan a mayores valores de Hto y menores pesos.

A partir de tomar como punto crítico el valor de Hto $\leq 22 \%$ debieron ser tratados con antiparasitarios 9 individuos para el grupo TRAT s/L y un solo animal para el grupo TRAT c/L. Asimismo se observaron mayores valores de HPG para el grupo de animales que no consumían Leucaena y menores valores de las demás variables (Hto y peso) en todas las mediciones.

En conclusión, bajo las condiciones de la presente experiencia, los resultados demuestran que el consumo de Leucaena repercutió favorablemente en la disminución del recuento de HPG. La mejor evolución del peso vivo y del volumen globular observado en los terneros alientan la realización de futuras experiencias para un mayor conocimiento de esta alternativa, que augura un importante potencial como parte de un sistema de control parasitario.

Agradecimientos. A Sr. César Díaz y esposa, por permitirnos realizar el ensayo en su campo. Por colaborar en las actividades, a Karina Nenning, Randolf Terrazas, Rodolfo Casco, Gustavo Casadei, Juana Jiménez, Eduardo Alberto y Hugo Fretes. Al Ministerio de Producción de Formosa, por facilitar la balanza utilizada.

\section{REFERENCIAS}

1. Aerts RJ, Barry TN, McNabb WC. 1999. Polyphenols and agriculture: beneficial effects of proanthocyanidins in forages. Agricult Ecosyst \& Environ 75: 1-12.

2. Aguirre DH, Cafrune MM, Viñabal AE, Salatín AO. 2002. Aspectos epidemiológicos y terapéuticos de la nematodiasis gastrointestinal caprina en un área subtropical de Argentina. Rev Investig Agropec 31: 25-40. 
3. Anziani OS. 2013. Anthelmintic resistance in nematodes of herbivores hosts in Argentina. Proceed 24th Intern Conf World Ass Advan Vet Parasitology (Perth, Australia). http://www.scielo.org.ar/img/revistas/ria/v41n1/html/v41n1a06.htm

4. Anziani OS, Fiel CA. 2015. Resistencia a los antihelmínticos en nematodos que parasitan a los rumiantes en la Argentina. Rev Investig Agrop (RIA) 41: 34-46.

5. Araujo SE, McManus C, Amarante AF, Verdolin V, Louvandini H. 2009. Nematódeos de ruminantes em pastagem com diferentes sistemas de pastejo com ovinos e bovinos. Pesq Agropec Bras 44: 1191-1197.

6. Athanasiadou S, Kyriazakis I, Jakson F, Coop RL. 2000. Consequences of longterm feeding with condensed tannins on sheep parasitised with Trichostrongylus colubriformis. Intern J Parasitol 30: 1025-1033.

7. Athanasiadou S, Kyriazakis I, Jackson F, Coop RL. 2001. Direct anthelmintic effects of condensed tannins towards different gastrointestinal nematodes of sheep: in vitro and in vivo studies. Vet Parasitol 99: 205-219.

8. Caracostantogolo $\mathbf{J}$ et al. 2005. Evaluación de la resistencia a los antihelmínticos en rumiantes en Argentina. En: Producción y Sanidad Animal, FAO, Roma 2005.

9. Corticelli B, Lai M. 1963. Ricerche sulla tecnica di coltura delle larve infestive degli strongili gastro-intestinali del bovino. Acta Med Vet 9 : V-VI.

10. Hammond JA, Fielding D, Bishop SC. 1997. Prospects for plant anthelmintics in tropical veterinary medicine. Vet Res Comm 21: 213.

11. Haslam E, Lilley TH, Cai Y, Martin R, Mangnolato D. 1989. Traditional herbal medicines. The role of polyphenols. Planta Médica 55: 1-8.

12. Hoste H, Jackson F, Athanasiadou S, Thamsborg SM, Hoskin SO. 2006. The effects of tannin-rich plants on parasitic nematodes in ruminants. Trends in Parasitol 22: 253-261.

13. Jean BC. 1998. Aspectes nutritionnels et toxicologiques des tannins. Rev Méd Vét 149: 911-920.

14. Kenyon F et al. 2009. The role of targeted selective treatments in the development of refugia based approaches to the control of gastrointestinal nematodes of small ruminants. Vet Parasitol 164: 3-11.

15. Lobayan SI, Schapiro JH, Fiel CA, Zabalo MM, Roselli JG. 2017. Resistencia a los antihelmínticos en bovinos del nordeste de Corrientes (Argentina). Rev vet 28: 138-140.

16. López CJ et al. 2015. Efecto antihelmíntico in vitro de extractos vegetales en nematodos gastrointestinales de ovinos de pelo. Prod Agrop \& Desarr Sost 4: 11-25.

17. Mederos A, Banchero G. 2013. Parasitosis gastrointestinales de ovinos y bovinos. http://www.produccion.animal.com.ar/sanidad_intoxicaciones_metabolicos/parasit arias/parasitarias_ovinos /21-gastrointestinales_avances.pdf.

18. Morales G, Pino LA, Sandoval E, Jiménez D, Morales J. 2012. Relación entre la condición corporal y el nivel de infestación parasitaria en bovinos a pastoreo como criterio para el tratamiento antihelmíntico selectivo. Rev Investig Vet 23: 80-89.

19. Morgan ER, Cavill L, Curry GE, Word RM, Mitchell ES. 2005. Effects of aggregation and sample size on composite faecal egg counts in sheep. Vet Parasitol 131: 79-87.

20. Niezen JH et al. 2002. The effect of feeding sulla (Hedysarum coronarium) or lucerne (Medicago sativa) on lamb parasite burdens and development of immunity to gastrointestinal nematodes. Vet Parasitol 105: 229-245.

21. Oliveira L et al. 2011. Effect of six tropical tanniferous plant extracts on larval exsheathment of Haemonchus contortus. Rev Bras Parasit Vet 20: 155-160. 
22. OMS. 2000. Evaluación de residuos de ciertos fármacos de uso veterinario en los alimentos. Disponible en [whqlibdoc.who.int/trs/WHO_TRS_888_spa.pdf ]

23. Orozco M, Álvarez V, Jiménez A, Acuña O. 2009. Evaluación in vitro de hongos nematófagos para el control biológico de nematodos gastrointestinales de rumiantes. Rev MVZ (Córdoba, Colombia), 14: 3.

24. Pachas N. 2011. Leucaena, un arbolito que se las trae. Rev Prod XXI 238: 20-26.

25. Roberts F, O'Sullivan PJ. 1949. Methods for egg counts and larval cultures for strongyles infesting the gastrointestinal tract of cattle. Aust J Agric Res 24: 947-953.

26. Sangster NC, Gill J. 1999. Pharmacology of anthelmintic resistance. Parasitlol Today $15: 141-146$.

27. Soca M. 2006. La agroforestería y taninos condensados, una estrategia para el control de las parasitosis de los pequeños rumiantes. On line: www.avpa.ula.ve/docu PDFs/conferencias/agroforesteria.pdf

28. Suárez V, Olaechea F, Rossanigo C, Romero J. 2007. Enfermedades parasitarias de los ovinos y otros rumiantes menores en el cono sur de América. On line: http://helminto.inta.gob.ar/Alumnos/Libro\%20parasitosis\%20ovinas.\%20Su\%C3\% A1erz\%20et\%20al.\%202007.pdf

29. Vargas RC. 2006. Control de haemonchosis en caprinos (método Famacha). Agronomía Mesoamericana. http://www.redalyc.org/articulo.oa?id=43717112> ISSN

30. Waghorn GC, McNabb WC. 2003. Consequences of plant phenolic compounds for productivity and health of ruminants. Proceed Nutr Soc 62: 383-392. 